\title{
Perfil da clientela estomizada residente no município de Ponte Nova, Minas Gerais
}

\author{
Profile of the ostomized patients resident in the city of Ponte Nova, \\ Minas Gerais, Brazil
}

\author{
RAFAELA MAGALHÃES FERNANDES ${ }^{1}$, ELINE LIMA BORGES MIGUIR ${ }^{2}$, TEREZINHA VIECCELLI DONOSO ${ }^{3}$
}

${ }^{1}$ Enfermeira do Departamento de Medicina e Enfermagem da Universidade Federal de Viçosa (UFV); Especialista em Saúde Coletiva. ${ }^{2}$ Doutora em Enfermagem; Professora Adjunta da Escola de Enfermagem da Universidade Federal de Minas Gerais (UFMG); Enfermeira estomaterapeuta da Associação Brasileira de Estomaterapia (SOBEST). ${ }^{3}$ Mestre em Enfermagem e doutora em Ciências da Saúde; Enfermeira. Professora adjunta da Escola de Enfermagem da UFMG.

FERNANDES RM; MIGUIR ELB; DONOSO TV. Perfil da clientela estomizada residente no município de Ponte Nova, Minas Gerais. Rev bras Coloproct, 2011;30(4): 385-392.

RESUMO: Um estoma pode ser um sério limitador da qualidade de vida de pessoas obrigadas a conviver com essa condição. Conhecer os aspectos demográficos e clínicos de pessoas assistidas por programas de atenção ao estomizado é essencial para o estabelecimento de protocolos assistenciais visando à melhoria do cuidado prestado. Trata-se de estudo descritivo, visando caracterizar os pacientes estomizados residentes em uma cidade de Minas Gerais e inseridos no Programa de Atenção à Pessoa Ostomizada do local, no período de 1994 a 2006. A amostra foi constituída por 12 pacientes, todos colostomizados, com diagnóstico inicial, em ordem de ocorrência, de câncer de reto, de fístula vesicorretal e de câncer pélvico com acometimento das alças intestinais. Complicações no estoma ou na pele ao redor deste foram observadas em $66,1 \%$ da amostra, e 58,30\% dos pacientes estavam estomizados há dois anos ou mais. Os pacientes relataram alterações nos aspectos físicos e emocionais após a realização do estoma, interferindo em sua qualidade de vida. Constatou-se, porém, que para a maioria dos pacientes, o programa é considerado apenas um centro de concessão de dispositivos. Os mesmos desconhecem o papel da equipe multidisciplinar na recuperação e reabilitação precoces.

Descritores: Colostomia. Qualidade de vida. Reabilitação.

Escola de enfermagem da Universidade Federal de Minas Gerais (UFMG)

$\overline{\text { Recebido em 23/08/2010 }}$

Aceito para publicação em 24/09/2010 


\section{INTRODUÇÃO}

O paciente portador de estoma intestinal ou urinário pode sentir-se diferente, em uma sociedade na qual os tabus em relação ao corpo são frequentes, principalmente quando relacionados às partes íntimas. Em geral, evita-se falar abertamente sobre esses órgãos, e não são raras as situações em que, em função desses tabus, os indivíduos protelam até o limite do tolerável à procura de assistência médica, recorrendo muitas vezes a tratamentos paliativos e a automedicação.

Para o paciente ostomizado, a mutilação do corpo e o uso do dispositivo coletor levam-no a necessidade de reconstruir sua identidade corporal. A imagem corporal é interpretada como sendo desfigurada ou em processo de reelaboração. A presença do estoma pode provocar o isolamento do indivíduo retirando-o do convívio social ${ }^{1}$.

O estoma resulta, geralmente, de uma cirurgia mutilante e traumatizante. Dessa forma, uma estomia pode ser um sério limitador da qualidade de vida, sendo que os pacientes estomizados enfrentam dificuldades orgânicas e emocionais ${ }^{2}$.

O vocábulo estoma é de origem grega e compreende a abertura de uma boca visando à exteriorização de qualquer víscera oca existente no corpo. Essa abertura é realizada cirurgicamente e tem por objetivo promover a eliminação de efluentes, tais como fezes e urina para o meio externo ${ }^{3}$, sendo, portanto, denominado estoma de eliminação.

A nomenclatura utilizada para definir o tipo de estoma realizado advém do segmento exteriorizado. Os estomas urinários são comumente denominados de derivações urinárias e são realizados em pacientes portadores de doenças que envolvem a pelve renal, ureteres, bexiga e uretra, com o objetivo de preservar a função renal. O estoma intestinal, por sua vez, é indicado quando alguma parte do intestino apresenta disfunção, obstrução ou lesão, podendo ser ileostomia, cecostomia ou colostomia.

Para facilitar e auxiliar a reabilitação da pessoa estomizada são oferecidos diversos produtos, dentre os quais se destacam os equipamentos coletores e adjuvantes de proteção de pele que possibilitam maior conforto e melhor qualidade de vida aos estomizados. Além do uso do equipamento correto, o estomizado requer um atendimento individualizado devido às transformações radicais ocorridas em sua vida.
Para o enfrentamento dessa situação no contexto social, algumas medidas alternativas são propostas. É fundamental o desenvolvimento de um trabalho interdisciplinar, envolvendo todos os profissionais comprometidos com a assistência do paciente, para favorecer a aceitação da sua nova imagem corporal, propiciar a realização do autocuidado e promover a reabilitação ${ }^{2}$.

No Brasil, até meados dos anos 1970, as questões relacionadas às pessoas com estoma eram abordadas apenas por alguns profissionais com habilidades nessa área, geralmente médicos. Somente na década de 1980, com o trabalho de lideranças das associações de estomizados e de outros profissionais envolvidos nessa causa é que foram criados, na Constituição Brasileira de 1988, capítulos e artigos específicos que tratam dos direitos do cidadão e dos deveres do Estado, em relação à pessoa portadora de deficiência, inclusive portadores de estoma.

O Ministério da Saúde, por meio da portaria n. 400 de 16 de novembro de 2009 estabelece diretrizes nacionais para a Atenção à Saúde de Pessoas Ostomizadas, no âmbito do Sistema Único de Saúde (SUS) ${ }^{4}$. A finalidade dessa portaria é atender a todos os pacientes portadores de estomas urinários e intestinais que apresentem indicação para concessão de dispositivos coletores. Para tanto, o paciente beneficiário deve estar cadastrado em um dos centros de referência de algum programa e ser submetido à avaliação clínica por profissionais médicos e enfermeiros.

Um desses centros de referência encontra-se localizado na macrorregião Leste do Estado de Minas Gerais, mais precisamente no município de Ponte Nova. Essa Unidade atende a todos os pacientes dessa macrorregião e também os da microrregião de Viçosa. O atendimento aos pacientes iniciou em 1994, sendo que o programa se limitava unicamente à distribuição dos equipamentos coletores, não havendo, portanto, ações relacionadas à prestação de assistência a essa clientela. Esses equipamentos eram entregues na própria Secretaria de Saúde do município, e a equipe do programa era composta somente por auxiliares administrativos.

Em 2009, o atendimento passou a ser realizado pela equipe de enfermagem do programa e a avaliação do estoma e a indicação do equipamento coletor tornaram-se responsabilidade do enfermeiro.

Conhecer os aspectos demográficos e clínicos dos pacientes estomizados atendidos pelo programa 
é essencial para o estabelecimento de protocolos assistenciais visando à melhoria do cuidado prestado, uma vez que a informação existente é escassa e pouco divulgada. Nos prontuários não há dados referentes à evolução clínica, sendo a informação registrada apenas na ficha de cadastro que é preenchida no momento da inclusão da pessoa estomizada no programa. Os dados existentes são insuficientes para a caracterização da clientela em questão. Dessa forma, não há informações relacionadas à qualidade de vida desses pacientes. Não se sabe qual a opinião dessas pessoas quanto à assistência prestada pelo programa e sobre quais são as suas reais necessidades.

Diante da escassez de informações sobre a clientela atendida e a lacuna de conhecimento das reais necessidades desses estomizados, foi desenvolvido este estudo. Acredita-se que o resultado poderá subsidiar a sistematização da assistência e a elaboração de protocolos assistenciais visando à reabilitação precoce da clientela residente em Ponte Nova, Minas Gerais.

\section{OBJETIVO}

Caracterizar a clientela estomizada residente em Ponte Nova, Minas Gerais.

\section{METODOLOGIA}

Trata-se de uma pesquisa descritiva, realizada com os pacientes portadores de estoma intestinal e urinário residentes em Ponte Nova e cadastrados no Programa de Atenção à Pessoa Ostomizada das microrregiões de Ponte Nova e Viçosa, Minas Gerais, no período de 1994 a 2006. Durante a coleta de dados foram identificados 25 pacientes. Destes, quatro submeteram-se à reconstituição de trânsito, quatro faleceram, quatro não foram localizados e um havia mudado para outro município. Dessa forma, a amostra foi constituída por 12 pacientes.

O projeto de pesquisa foi aprovado pelo Comitê de Ética em Pesquisa da Universidade Federal de Minas Gerais (COEP-UFMG) sob o parecer número ETIC 380/07 e pela Secretaria Municipal de Saúde de Ponte Nova, em 12 de março de 2007. Todos os pacientes estomizados concordaram em participar deste estudo e assinaram o termo de consentimento livre e esclarecido. Foi garantido aos participantes o anonimato e a isenção total de riscos.
Os pacientes foram submetidos a consultas do enfermeiro, o que foi agendado previamente por telefone e, para aqueles impossibilitados de comparecer à unidade devido a problemas de saúde, foram realizadas visitas domiciliares. A avaliação clínica dos pacientes foi realizada em consultório apropriado.

Para facilitar os registros de coleta de dados, foi elaborado um instrumento com questões referentes a aspectos sociodemográficos, características do estoma e do efluente e suas complicações, autocuidado realizado e reabilitação. O local de abordagem dos pacientes foi a Unidade do Programa de Saúde da Família (PSF), localizada na área central do Município de Ponte Nova, Minas Gerais, local de funcionamento do Programa de Atenção à Pessoa Ostomizada.

Os dados obtidos foram tabulados e organizados em gráficos e tabelas por meio do programa Excel, versão 2003, seguido de análise descritiva. Os resultados foram analisados e discutidos à luz da literatura específica.

\section{RESULTADOS}

Todos os pacientes que compuseram a amostra eram adultos e portadores de estoma intestinal, sendo a maioria $(66,7 \%)$ mulheres. A distribuição conforme faixa etária encontra-se no Gráfico 1.

A idade dos pacientes variou de 27 a 81 anos, com média de 61,6 anos, sendo a maioria $(66,6 \%)$ idosa. Destaca-se a ausência de pacientes na faixa dos 40 a 49 anos.

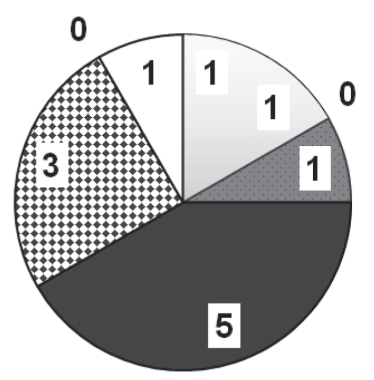

옹 25 a 29 anos

目 30 a 39 anos

$\square 40$ a 49 anos

$\square 50$ a 59 anos

-60 a 69 anos

日70 a 79 anos

$\square 80$ anos ou mais

Gráfico 1 - Perfil da clientela estomizada residente no município de Ponte Nova, Minas Gerais. Distribuição dos pacientes portadores de estoma residentes em Ponte Nova, segundo faixa etária. Fonte: Instituto Brasileiro de Geografia e Estatística (IBGE). Belo Horizonte, 2007. 
Quanto à situação conjugal, quase metade dos pacientes $(41,7 \%)$ estava casada. Cinco pacientes $(41,7 \%)$ estavam viúvos, um $(8,3 \%)$ divorciado e um $(8,3 \%)$ solteiro.

Para avaliação da raça utilizou-se o critério preconizado pelo Instituto Brasileiro de Geografia e Estatística (IBGE). Nove pacientes $(75,0 \%)$ pertenciam à raça branca. A raça negra e a parda estavam representadas por três pacientes $(25,0 \%)$.

Neste estudo, a renda salarial dos pacientes estomizados foi investigada e expressa em salários mínimos. Todos os pacientes tinham renda mensal inferior ou igual a três salários mínimos, sendo que seis $(50,0 \%)$ recebiam um salário mínimo, cinco $(41,7 \%)$ de dois a três salários mínimos e um paciente $(8,3 \%)$ recebia menos de um salário mínimo.

Quanto à situação laboral citada pelos pacientes houve predomínio de trabalhadoras do lar $(33,3 \%)$, empregadas domésticas $(16,7 \%)$ e vendedores $(16,7 \%)$. As demais profissões ou ocupações citadas foram professor, costureira, barbeiro e servidor público, com um representante cada. Destaca-se que apenas um paciente $(8,3 \%)$ exercia atividade fora do lar, sendo os demais pensionistas ou aposentados.

Todos os pacientes eram portadores de colostomia definitiva ou temporária, sendo que em 11 pacientes $(91,7 \%)$, esta era em cólon descendente ou sigmoide e em um $(8,3 \%)$ em cólon ascendente.

A maioria $(75,0 \%)$ dos pacientes necessitou de estoma devido à neoplasia maligna que acometia o reto em oito pacientes $(66,7 \%)$ e com comprometimento de alças intestinais em um paciente $(8,3 \%)$. Os demais $(25 \%)$ apresentavam fístula vesicorretal decorrente de doença inflamatória.

$\mathrm{O}$ exame físico possibilitou caracterizar os pacientes quanto ao estado geral e à capacidade de locomoção. Dez pacientes $(83,3 \%)$ apresentavam estado geral bom e dois $(16,7 \%)$ encontravam-se em estado geral regular. Três pacientes $(25 \%)$ apresentavam capacidade de locomoção prejudicada, sendo que dois faziam uso de órtese e um $(8,3 \%)$ era acamado devido a quadro de paraplegia causado por acidente automobilístico.

A maioria dos pacientes (83\%) apresentava agravos crônicos, sendo mais frequentes o diabetes tipo 2, insuficiência renal, alcoolismo, doença de Crohn e paraplegia.
Quanto ao tempo de convivência com o estoma, menos da metade $(41,6 \%)$ o possuíam a menos de um ano, podendo ser considerados como em fase de adaptação. Houve predomínio de pacientes estomizados há dois anos ou mais $(58,30 \%)$, sendo que dois pacientes eram estomizados há mais de 16 anos.

A avaliação do estoma permitiu constatar que a maioria $(91,6 \%)$ foi exteriorizada através do músculo reto-abdominal. O diâmetro e a protrusão dos estomas variavam de 22 a $40 \mathrm{~mm}$ e de $1 \mathrm{~cm}$ e mais, respectivamente.

A maioria $(66,7 \%)$ dos pacientes apresentava complicações no estoma ou na pele ao redor deste. Em alguns pacientes havia mais de uma complicação. No grupo dos oito pacientes com complicações houve predomínio de dermatite $(50,0 \%)$, prolapso $(37,5 \%)$ e hérnia periestomal, esta última estava em um paciente.

A maioria dos estomas apresentava aspecto fisiológico, mas em dois casos (16,6\%) observou-se edema acentuado. Entretanto, em $75,0 \%$ dos pacientes, o estoma era plano.

O efluente era de consistência pastosa ou semipastosa na maioria $(91,7 \%)$ dos pacientes. Esse dado é compatível com a localização do estoma, de predomínio em cólon esquerdo. Apenas um paciente apresentava efluente líquido, sendo que neste o estoma era no cólon direito.

Outra variável avaliada foi o uso de equipamento coletor adequado. Para tal, consideraram-se as características do estoma e do efluente, a área para fixação do equipamento e o estilo de vida dos pacientes.

A maioria dos pacientes fazia uso do equipamento coletor drenável ou aberto $(66,6 \%)$, sistema de uma peça $(91,6 \%)$ e recortável $(91,6 \%)$. Constatou-se que todos os pacientes usavam o dispositivo adequado para as condições e características apresentadas.

O padrão de troca do equipamento foi de uma a duas vezes por dia para aqueles que usavam coletor não-drenável ou fechado e de duas a quatro vezes por semana para os que utilizam o sistema drenável ou aberto. Dois pacientes também faziam uso de adjuvantes (pasta de resina sintética) para auxiliar a fixação do equipamento fornecido pelo programa, pois apresentavam irregularidades na pele.

Quanto ao autocuidado relacionado à habilidade para realizar as trocas do equipamento, constatou-se que oito pacientes $(66,7 \%)$ possuíam habilidade to- 
tal, trocando os equipamentos sozinhos, um $(8,3 \%)$ possuía habilidade parcial, necessitando de ajuda de familiar e em três pacientes $(25,0 \%)$ o procedimento era totalmente realizado por familiares. Nesses casos, observou-se que os familiares demonstravam dedicação e disposição para o cuidado e conhecimento sobre o tema.

Neste estudo, 58,3\% dos pacientes descreveram que as alterações orgânicas decorrentes da confecção do estoma afetaram a sexualidade e o padrão de sono. O primeiro aspecto, conforme $33,3 \%$ dos pacientes, devia-se mais à baixa autoestima do que à limitação física. A alteração do sono foi relacionada à presença do estoma, principalmente nos primeiros meses após a cirurgia e ao medo acerca do futuro.

No que tange às expectativas dos pacientes em relação ao programa, $33,3 \%$ relataram que nem todas as necessidades eram atendidas.

\section{DISCUSSÃO}

Os profissionais de saúde devem favorecer o desenvolvimento do autocuidado dos pacientes estomizados para que estes consigam alcançar a reabilitação. Identificar e entender as peculiaridades de cada um faz parte desse processo.

Constatou-se neste estudo o predomínio de pacientes com mais de 60 anos, uma vez que a maior incidência das neoplasias ocorre em pessoas com idade superior a 40 anos, característica essa tida não só como fator de risco para o aparecimento dos cânceres, mas também como determinante para os tratamentos cirúrgicos geradores de estoma ${ }^{5}$.

$\mathrm{O}$ agravo mais frequente para o desencadeamento do estoma foi o câncer de reto. Esses dados são corroborados por outros autores, no que se refere às colostomias definitivas, as quais são geralmente realizadas quando o paciente apresenta diagnóstico de neoplasia de reto e ânus ${ }^{6}$. Segundo o Ministério da Saúde ${ }^{7}$, conforme dados divulgados em 2001, a incidência desse tipo de câncer no Brasil é de 10,4 em homens e 8,9 em mulheres para cada 100 mil habitantes. Alguns autores ressaltam a importância do contínuo investimento na divulgação e implementação de medidas preventivas, incluindo a realização de exames para o diagnóstico precoce da doença naquelas pessoas com fator de risco, a fim de evitar e diminuir a necessidade de realiza- ção de estomas ${ }^{8,9}$.

Todos os pacientes apresentavam colostomia do cólon ascendente, descendente ou sigmoide. Essa intervenção geralmente é realizada quando o paciente é submetido a procedimento cirúrgico decorrente de neoplasia intestinal. $\mathrm{O}$ carcinoma de tipo intestinal é o principal tipo de tumor encontrado em populações de alto risco, acomete mais homens que mulheres, com faixa etária em torno dos $60 \operatorname{anos}^{10}$.

O risco para o desenvolvimento de câncer colorretal (CCR) pode ser classificado em baixo risco, risco moderado e alto risco. Na primeira categoria encontram-se pacientes com idade superior a 50 anos e sem outros fatores de risco para CCR. Na segunda estão os pacientes com histórico familiar de CCR em pelo menos um parente de primeiro grau, história pessoal de pólipo superior a um centímetro ou múltiplos pólipos (independentes de tamanho) ou indivíduos com antecedente familiar pessoal de CCR tratados de forma curativa. Na categoria de alto risco estão os indivíduos com história familiar de CCR hereditário na forma de polipose adenomatosa familiar ou CCR hereditário sem polipose ou com diagnóstico de doença inflamatória intestinal na forma de pancolite ou colite esquerda ${ }^{11}$.

Quanto aos carcinomas de ânus e reto, a amputação desses segmentos foi, até pouco tempo, o tratamento de escolha para os carcinomas de canal anal. Nesse tipo de abordagem fazia-se a retirada do ânus e do reto e o procedimento terminava com uma colostomia definitiva ${ }^{12}$.

Em relação ao gênero, estudo que caracterizava esse dado na clientela estomizada constatou, em alguns momentos, o predomínio de mulheres nas casuísticas e, em outros momentos, o predomínio de homens, sem, no entanto detectar análise de significância estatística $^{8}$. O predomínio de mulheres no grupo em questão pode estar relacionado às características da doença de base, uma vez que o Instituto Nacional do Câncer (INCA) informou que no período de 1995 a 1999, o câncer de cólon e reto se constituiu como a quarta causa de morte entre mulheres por câncer no país, perdendo apenas para os cânceres de mama, pulmões e estômago ${ }^{13}$. Lembra-se que o gênero do estomizado pode influenciar na sua adaptação social. As mulheres tendem a demandar menos tempo para a reabilitação, embora demonstrem significantes graus de desespero, depressão e medo no período pré-opera- 
tório. Os homens, principalmente aqueles que desenvolvem impotência sexual, demoram um tempo maior para responder de forma satisfatória às atividades rotineiras, inclusive apresentam dificuldades mais acentuadas para o autocuidado 5 .

Quanto à situação conjugal, a análise desse dado fez-se importante porque essa variável, bem como a atividade sexual está diretamente relacionada aos problemas resultantes do estoma ${ }^{14}$. Constatou-se que $41,6 \%$ mantinham-se casados.

Os pacientes que manifestaram alteração dos hábitos sexuais relacionaram essa situação à baixa autoestima como fator desencadeante, se sobrepondo à limitação física. Pesquisa qualitativa sobre sexualidade dos estomizados revela que, tanto o sujeito portador de colostomia como seu parceiro sexual carecem de mais informações a respeito de sua sexualidade ${ }^{15}$. Constata também que os profissionais da saúde necessitam de preparo específico no sentido de atender aos questionamentos concernentes à sexualidade, sobretudo com referência aos sujeitos portadores de colostomia.

Ressalta-se que não foram encontrados estudos na literatura científica em que se avaliem os pacientes estomizados quanto à raça. No entanto, em pesquisa sobre incidência de CCR, esse agravo foi mais frequente em indivíduos brancos, seguidos de pardos e negros respectivamente ${ }^{9}$, dados que se assemelham aos encontrados neste estudo.

A situação socioeconômica pode interferir na qualidade de vida do estomizado ${ }^{16}$. Todos os pacientes investigados tinham renda mensal igual ou inferior a três salários mínimos. Essa questão é inerente inclusive a possibilidade ou a dificuldade para se adquirir os equipamentos e adjuvantes necessários para uma boa qualidade de vida, quando alguns desses artigos não são fornecidos pelos programas de assistência ao estomizado.

Importante destacar que apenas um dos pacientes estudados exercia a sua profissão. Geralmente, as pessoas estomizadas têm grandes dificuldades para retornar ao trabalho, pois se sentem inseguras para continuar cuidando da estomia e ainda trabalhar. A ausência de atividade laborativa pode levá-las à ociosidade e ao isolamento social ${ }^{17}$.

Além dos estomas, os pacientes em questão apresentavam agravos como depressão, hipertensão, alcoolismo e doença de Crohn. Porém, todos os entrevis- tados informaram realizar avaliação clínica periódica para controle do agravo. Verificou-se, inclusive, que muitos faziam essa avaliação com o médico particular, utilizando-se do programa apenas para a busca dos equipamentos e adjuvantes. Esse fato, associado aos casos dos pacientes que recorrem aos familiares para buscarem os equipamentos, podem interferir na assistência prestada pela equipe dos profissionais do programa, uma vez que se torna impossível acompanhar a evolução e estabelecer novas condutas que visem também ao controle de agravos paralelos.

Outro fator observado e que também dificulta a assistência é a não-realização de visitas domiciliares aos pacientes acamados, devido à falta de sistematização e de condições do programa, por exemplo, a inexistência de automóvel disponível para a realização das visitas. Os profissionais são dependentes da disponibilidade de veículos pertencentes à Secretaria de Saúde ou da ambulância do Município. Esse problema poderia ser minimizado por meio da assistência da equipe multiprofissional do Programa de Saúde da Família (PSF) no domicílio desses pacientes assegurando continuidade da assistência e intervenção nas complicações.

Quanto ao tempo de convivência com o estoma, observou-se que há pacientes no programa que foram estomizados antes de 1994. Ainda que a convivência com a condição de estomizado tenha se iniciado há muito tempo, a reabilitação para algumas pessoas se caracteriza como um processo longo e contínuo ${ }^{18}$. Os serviços que prestam assistência a essa clientela devem contar com equipe multidisciplinar visando a um atendimento holístico, individualizado e, principalmente, contínuo.

À avaliação do estado geral, constatou-se que a maioria dos pacientes apresentava-se em bom estado. Essa avaliação foi subjetiva e baseada no conjunto de dados exibidos pelo paciente. Para os pacientes estomizados, a avaliação do estado geral subsidia a equipe de saúde na escolha de estratégias para a orientação do autocuidado e às condutas que visem à melhor qualidade de vida.

Quanto às complicações, é sabido que alguns fatores de ordem geral podem favorecer o aparecimento de complicações do estoma como idade avançada, desnutrição e radiação pré e pós-operatórias ${ }^{6}$.

O surgimento da hérnia paraestomal é uma con- 
sequência direta da confecção do estoma. Apesar de todos os esforços empregados na sua prevenção, a frequência é bastante elevada e aumenta com o tempo ${ }^{19}$. Neste estudo, verificou-se que o paciente que apresentava hérnia paraestomal possuía alguns fatores predisponentes para o seu desenvolvimento, tais como obesidade e sedentarismo ${ }^{20}$. Embora tenha sido proposta a correção cirúrgica, a família optou por manter a hérnia paraestomal por receio de alterar a qualidade de vida do paciente.

Embora possa ocorrer prolapso, essa não é uma complicação comum em pacientes estomizados ${ }^{21}$. Nos pacientes que apresentavam prolapso, observou-se que estes eram pequenos, sem a presença de outras complicações e sem comprometimento do cuidado com o estoma e uso do equipamento e adjuvantes.

Concernente às lesões periestomais, observou-se pacientes com dermatite. Essas lesões geralmente decorrem do uso inadequado dos equipamentos coletores, mais precisamente pelo corte excessivo do orifício da barreira protetora em relação ao estoma, deixando a pele exposta à ação do efluente, ou por indicação inadequada do equipamento ao tipo de estoma ${ }^{8}$. Equipamentos coletores e adjuvantes existentes no mercado devem ser apresentados nos mínimos detalhes, aos pacientes estomizados. O equipamento utilizado em alguns serviços é recomendado mediante resultado da avaliação realizada no momento, mas, com o passar do tempo, pode ser substituído, daí a necessidade de avaliação contínua.

Quanto às características do efluente, pode-se inferir que o achado encontrado é compatível com o tipo de estoma apresentado pelos pacientes, uma vez que a maioria apresentava colostomia esquerda.

Para garantir a reabilitação faz-se importante minimizar o medo e a insegurança do paciente quanto ao risco de vazamento do equipamento coletor. Para isso, não há necessidade de se padronizar o número de trocas do equipamento, uma vez que a frequência de trocas é individualizada, devendo-se respeitar as características do efluente, do estoma e da pele do indivíduo. Destaca-se que a troca deve ser realizada antes da ocorrência de vazamentos.

Em relação à habilidade para a troca do equipamento, os pacientes que necessitavam da ajuda de fa- miliares para realizá-la justificaram essa necessidade devido à acuidade visual diminuída ou à deficiência motora em membros superiores. Relembra-se que, atualmente, é possível encontrar uma diversidade de placas e bolsas coletoras, que visam adaptar-se cada vez mais às necessidades da pessoa estomizada, favorecendo o autocuidado ${ }^{22}$.

\section{CONSIDERAÇÕES FINAIS}

A assistência voltada ao paciente estomizado vai além da compreensão específica do cuidado com o estoma, tornando-se necessária uma reorganização da assistência prestada a essas pessoas com o objetivo de alcançar os princípios da universalidade, equidade e integralidade.

Os pacientes estomizados residentes em Ponte Nova eram portadores de colostomia, decorrente de intervenção cirúrgica demandada por câncer de reto ou câncer com acometimento de alças intestinais e fístulas por doença inflamatória intestinal. A maioria era constituída por mulheres e a idade variou de 27 a 81 anos, com média de 61,6 anos. O tempo de convivência com o estoma variou de alguns meses a mais de 16 anos.

A complicação mais comum apresentada pelos pacientes foi a dermatite periestomal. Alguns pacientes apresentavam prolapso, porém todos de pequeno tamanho.

Percebe-se que, para a maioria dos pacientes envolvidos neste estudo, o Programa de Atenção à Pessoa Ostomizada é considerado apenas um centro de concessão de equipamentos e adjuvantes. Os pacientes desconhecem o papel da equipe multidisciplinar na recuperação e reabilitação precoces. Alguns pacientes sugeriram que fossem realizadas oficinas educativas ou grupos para a troca de experiência e a ampliação da equipe multidisciplinar com a inserção de nutricionista, assistente social e psicólogo. O trabalho em grupo, onde ocorre a troca de experiências, favorece ao enfrentamento das dificuldades que surgem, contribuindo para melhor aceitação e uma melhoria da qualidade de vida do paciente estomizado. 


\begin{abstract}
A stoma can be a serious limiting factor in the quality of life of people forced to live with it. Knowing the demographic and clinical characteristics of people assisted by ostomate care programs is essential for the establishment of protocols which focus on improving the care provided. This is a descriptive study and its purpose was to characterize the ostomates who live in any city in Minas Gerais state, Brazil, and were treated in the local Ostomate Care Program Center between 1994 and 2006. The sample consisted of 12 colostomized patients, with an initial diagnosis of, in order of occurrence, rectal cancer, rectovesical fistula and pelvic cancer affecting the intestinal loop. Complications in the stoma or in the skin around it were observed in $66.1 \%$ of the sample, of which $58.3 \%$ had been ostomized for two years or more. Patients reported changes in physical and emotional aspects after the stoma, which interferes with their quality of life. It has been proven, however, that for most of them, the Program is considered only as a center where they get their devices. They do not know the role of the multidisciplinary team in the early recovery and rehabilitation.
\end{abstract}

Key words: Colostomy. Quality of life. Rehabilitation.

\section{REFERÊNCIAS}

1. Martelli ZB. Necessidades de aprendizagem da pessoa colostomizada. Dissertação de Mestrado. Ribeirão Preto, São Paulo: Universidade de São Paulo, 1983.

2. Bechara RN, Bechara MS, Queiroz HC, Oliveira RB, Mota RS, Secchin LSB, et al. Abordagem multidisciplinar do ostomizado. Rev bras Coloproct, 2005; 25(2):146-49.

3. Gemelli LMG, Zago MMF. A interpretação do cuidado com o ostomizado na visão do enfermeiro: um estudo de caso. Rev Latino-Am Enfermagem. 2002;10(1):34-40.

4. Brasil. Ministério da Saúde. Portaria $n^{\circ} 400$ de 16 de novembro de 2009. Diretrizes Nacionais para a Atenção à Saúde das Pessoas Ostomizadas. Secretaria de atenção à saúde; 2009.

5. Macedo MS, Nogueira LT, Luz MHBA. Perfil dos estomizados atendidos em hospital de referência em Teresina. Rev Estima. 2005;3(4):25-28.

6. Meirelles CA, Ferraz CA. Avaliação da qualidade do processo de demarcação do estoma intestinal e das intercorrências tardias em pacientes ostomizados. Rev Latino-Am de Enfermagem. 2001;9(5):32-8.

7. Brasil. Ministério da Saúde. Departamento de Atenção à Saúde. Brasília: Ministério da Saúde, 2006. 60 p. - (série a. normas e manuais técnicos) (série pactos pela saúde 2006, v.4).

8. Yamada BFA, Cesaretti IUR, Marcondes MGSG, Morais JF, Prado AAB. Ocorrência de complicações no estoma e pele periestoma: estudo retrospectivo. Revista Estima. 2003;1(3):16-24.

9. Mantovani MF, Turra K, Trencin C. Assistindo o adulto com ostomia na associação paranaense dos ostomizados. Rev Nursing. 2003;59(6):38-41.

10. Rodrigues LGM, Nogueira AMMF, Araújo LA, Salles PGO, Carvalho SP, Cabral MMDA. Metaplasia intestinal e carcinoma gástrico: correlação com os subtipos histológicos da neoplasia. J Bras Patol. 2001;37(4):279-86.

11. Habr-Gama A. Câncer coloretal: a importância de sua prevenção. Arq Gastroenterol. 2005;42(1):2-3.

12. Santos JR, Júlio César M. Câncer ano-retal-cólico - aspectos atuais: I - câncer anal. Rev Bras Coloproct. 2007;27(2):219-23.

13. Torres Neto J da R, Teixeira FR, Prudente ACL, Silvino CJ, Arciere JS, Vieira Filho MC. Estudo demográfico do câncer de cólon e reto no Estado de Sergipe. Rev Bras Coloproct. 2008;28(2):215-22.
14. Luz MHBA, Andrade D S, Amaral H O, Bezerra SMG, Benício CDAV, Leal ACA. Caracterização dos pacientes submetidos a estomas intestinais em um hospital público de Teresina-PI. Texto Contexto Enferm. 2009;18(1):140-6.

15. Freitas MRI, Pelá NTR. Subsídios para a compreensão da sexualidade do parceiro do sujeito portador de colostomia definitiva. Rev Latino-Am Enferm. 2000;8(5):28-33.

16. Violin MR, Mathias TAF, Uchimura TT. Perfil de clientes colostomizados inscritos em programa de atenção aos estomizados. Rev Eletr Enf. 2008;10(4):924-32. Disponível em: http://www.fen.ufg.br/revista/v10/n4/v10n4a05.htm. Acesso em 6 jul 2009.

17. Silva AL, Shimizu HE. O significado da mudança no modo de vida da pessoa com estomia intestinal definitiva. Rev Latino-Am Enferm. 2006;14(4):483-90.

18. United Ostomy Association Inc. Guia de Colostomia. 2004. Disponívelem:www.uoaa.org/ostomy-info/pubs/uoa-colostomyes.pdf. Acesso em 30 nov. 2009.

19. Carvalho CG de, Vale CEP, Castro Junior PC. Experiência inicial no tratamento das hérnias paraestomais. Rev bras Coloproct. 2008;28(2):251-56.

20. Carvalho CG, Vale CEP, Castro Júnior PC. Tratamento Cirúrgico da hérnia paraestomal por videolaparoscopia. Rev bras Coloproct. 2004;24(4):311-16.

21. Cruz GMG da, Constantino JRM, Chamone BC, Andrade MMA, Gomes DMBM. Complicações dos estomas em câncer colorretal: revisão de 21 complicações em 276 estomas realizados em 870 pacientes portadores de câncer colorretal. Rev bras Coloproct. 2008;28(1):50-60.

22. Caiscais AFMV, Martini JG, Almeida PJS. O impacto da ostomia no processo de viver humano. Texto Contexto Enferm. 2007;16(1):163-67.

Endereço para correspondência:

Eline Lima Borges

Escola de Enfermagem da Universidade Federal de Minas Gerais. MG Campus da Saúde

Avenida Alfredo Balena, 190, Sala 216 - CEP 30130.100.

Belo Horizonte - (MG), Brasil.

Telefax.: (31) 3409-9853

E-mail: eborges@ufmg.br 